\title{
SARS-CoV-2 infection induces robust germinal center CD4 T follicular helper cell responses in rhesus macaques
}

\section{Sonny Elizaldi}

University of California, Davis

Yashavanth Shaan Lakshmanappa

University of California, Davis https://orcid.org/0000-0002-7794-1963

Jamin Roh

University of California, Davis

\section{Brian Schmidt}

University of California, Davis

\section{Timothy Carroll}

University of California, Davis

\section{Kourtney Weaver}

Louisiana State University Health Sciences Center

Justin Smith

Louisiana State University Health Sciences Center https://orcid.org/0000-0001-7702-7920

Jesse Deere

University of California, Davis

Joseph Dutra

University of California, Davis

\section{Mars Stone}

Vitalant Research Institute

\section{Sergej Franz}

Rebecca Sammak

University of California, Davis

\section{Katherine Olstad}

University of California, Davis

\section{J. Rachel Reader}

University of California https://orcid.org/0000-0003-3864-6731

\section{Zhong-Min Ma}

University of California, Davis

\section{Nancy Nguyen}

University of California, Davis 


\section{Jennifer Watanabe}

University of California https://orcid.org/0000-0001-9408-3991

Jodie Usachenko

University of California, Davis

\section{Ramya Immareddy}

University of California, Davis

JoAnn Yee

University of California

\section{Daniela Weiskopf}

Division of Vaccine Discovery, La Jolla Institute for Allergy and Immunology, La Jolla, CA.

\section{Alessandro Sette}

La Jolla Institute for Immunology

\section{Dennis Hartigan-0'Connor}

University of California, Davis

\section{Stephen McSorley}

UC Davis

John Morrison

University of California

\section{Nam Tran}

University of California, Davis

\section{Graham Simmons}

Vitalant Research Institute

\section{Michael Busch}

University of California, San Francisco

\section{Pamela Kozlowsk}

Louisiana State University Health Sciences Center New Orleans

\section{Koen van Rompay}

UC Davis https://orcid.org/0000-0002-7375-1337

\section{Christopher Miller}

University of California, Davis

\section{Smita lyer ( $\nabla$ smiyer@ucdavis.edu )}

University of California Davis School of Veterinary Medicine and California National Primate Research Center.

\section{Article}

Keywords: SARS-CoV-2, Adaptive Immunity, Innate Immunity, SARS-CoV-2 specific antibody; SARS-CoV-2 specific T cells, Convalescent plasma, Nucleocapsid, Spike 
Posted Date: August 14th, 2020

DOl: https://doi.org/10.21203/rs.3.rs-51545/v1

License: (c) (i) This work is licensed under a Creative Commons Attribution 4.0 International License. Read Full License

Version of Record: A version of this preprint was published at Nature Communications on January 22nd, 2021. See the published version at https://doi.org/10.1038/s41467-020-20642-x. 


\section{Abstract}

CD4 $\mathrm{T}$ follicular helper $\left(\mathrm{T}_{\mathrm{fh}}\right)$ cells are important for the generation of durable and specific humoral protection against viral infections. The degree to which SARS-CoV-2 infection generates $T_{\text {fh }}$ cells and stimulates the germinal center response is an important question as we investigate vaccine options for the current pandemic. Here we report that SARS-CoV-2 infection resulted in transient accumulation of proinflammatory monocytes and proliferating $T_{\text {fh }}$ cells with a $T_{h} 1$ profile in peripheral blood. CD 4 helper cell responses were skewed predominantly toward $a T_{h} 1$ response in blood, lung, and lymph nodes. We observed the generation of germinal center $T_{\text {fh }}$ cells specific for the SARS-CoV-2 spike (S) and nucleocapsid $(\mathrm{N})$ proteins, and a corresponding early appearance of antiviral serum IgG antibodies. Our data suggest that a vaccine promoting $T_{h} 1$-type $T_{\text {fh }}$ responses that target the $S$ protein may lead to protective immunity.

\section{Introduction}

As of July 30th, 2020, SARS-CoV-2 has resulted in more than 17 million infections and more than half a million deaths, globally ${ }^{1}$. Unanticipated post-infection complications, such as multisystem inflammatory syndrome pose a serious threat ${ }^{2}$. An effective vaccine is paramount, and several SARS-CoV-2 vaccine candidates are in various phases of human testing worldwide ${ }^{3-5}$. The most effective vaccines induce antibodies that provide long-term protection ${ }^{6}$ and vaccines using attenuated virus elicit the most persistent antibody responses; therefore, understanding the immunological mechanisms characteristic of controlled SARS-CoV-2 infection is foundational to the selection of a vaccine capable of abating the pandemic 7,8 .

Generation of persistent immunity hinges on CD4 T follicular helper cells $\left(T_{f h}\right)$. We and others have demonstrated that peripheral CD4 $\mathrm{T}_{\text {fh }}$ cells predict antibody durability in the context of HIV and influenza vaccines ${ }^{9-11}$. While studies in humans demonstrate induction of $T_{\text {fh }}$ cells in COVID-19 patients ${ }^{12-14}$, the impact of SARS-CoV-2 infection on the generation of germinal center $\mathrm{T}_{\mathrm{fh}}$ cells is currently unknown. This is a detrimental gap in knowledge as understanding early correlates of durable antibodies, specifically those that circulate in peripheral blood, will aid in the ultimate selection of effective vaccine candidates. SARS-CoV-2-specific CD4 T cells responding to spike proteins have been observed in the peripheral blood of recovered patients ${ }^{15,16}$. Similar observations have been made with the 2002 SARS-CoV virus ${ }^{17,18}$, and studies in mouse models have demonstrated a critical role for CD4 T cells in viral clearance ${ }^{19}$. Together, these data emphasize the need to understand CD4 $T_{\text {fh }}$ responses following SARS-CoV-2 infection.

Because healthy rhesus macaques infected with SARS-CoV-2 resist immediate re-challenge with the virus 20-22, we hypothesized that understanding the CD4 $\mathrm{T}_{\text {fh }}$ and germinal center (GC) response following exposure to SARS-CoV-2 will provide a framework for understanding immune mechanisms of protection. Here we report that, following infection with SARS-CoV-2, adult rhesus macaques exhibited transient 
accumulation of activated, proliferating $T_{\text {fh }}$ cells with a $T_{h} 1$ profile in their peripheral blood. Perhaps more pertinent to SARS-CoV-2 as a respiratory virus, infection elicited robust GCs with SARS-CoV-2- reactive $T_{\text {fh }}$ cells within the mediastinal lymph nodes. The comprehensive immune analysis in a controlled animal model of mild diseases adds to our understanding of immune responses to SARS-CoV-2; the data suggest that vaccine platforms inducing $T_{h} 1-T_{\text {fh }}$ responses are likely to succeed in eliciting durable humoral responses.

\section{Results}

\section{Experimental Design}

To achieve our primary objective of assessing whether SARS-CoV-2 elicits $T_{\text {fh }}$ cells and germinal-center responses, we challenged eight adult rhesus macaques (4-5y, Table S1) with a high-dose of SARS-CoV-2 $\left(2 \times 10^{6} \mathrm{PFU}\right)$. Virus was administered via the intranasal, intratracheal, and ocular routes. Infection was monitored by following the quantity of viral RNA (VRNA) in nasal washes using qRT-PCR. Of the eight infected animals: four did not receive plasma infusion (Infected), two animals were infused with COVID19 convalescent human plasma 24 hours following inoculation $(I+C P)$, and two animals were infused with an identical volume of normal plasma lacking antibodies to SARS-CoV-2 (I+NP). (Fig. 1A). Pooled $\mathrm{CP}$ demonstrated a neutralizing titer of 1:1,149; but due to the estimated 50 -fold dilution after infusion (based on $4 \mathrm{ml} / \mathrm{kg}$ infusion volume and $~ 20 \%$ extracellular fluid), neutralizing activity in macaque sera 24 hours post infusion fell below the limit of detection (1:40) of the neutralization assay. Consistently, CP administration did not blunt acute viral loads and high vRNA levels were observed in all animals (Fig. 1B). Histopathological lesions of the lungs confirmed multifocal to locally extensive interstitial pneumonia of mild to moderate severity in infected animals (Figure S1A). However, these histological changes were not accompanied by fever, weight loss, or any other signs of clinical disease (Figure S1B). None of the animals developed acute respiratory distress. In summary, infection of healthy adult rhesus macaques with SARS-CoV-2 resulted in high viremia but generally produced no overt signs of clinical illness, providing a framework to investigate development of protective immune responses.

\section{SARS-CoV-2 infection leads to a rapid and transient shift in innate immune responses and increases the number of CD4 $\mathrm{T}$ follicular helper cells in peripheral blood.}

Evaluation of innate immune cell subsets in the peripheral blood (Fig. 1C) revealed no significant changes in either the proportion or absolute counts of neutrophils over time (Fig. 1D). However, rapid and divergent changes in specific myeloid cell subsets were observed. While CD14 + CD16 + pro-inflammatory monocytes significantly increased at Day 2 with a corresponding increase in CX3CR1 expression (Figure S1C), pDCs decreased in peripheral blood. We also noted a significant increase in myeloid DCs (mDC) within the infected group. Pro-inflammatory chemokines monocyte chemoattractant protein (MCP-1), interferon $y$-induced protein-10 (IP-10), interferon inducible T cell a chemoattractant (I-TAC) were significantly elevated at Day 2 and returned to baseline levels soon thereafter (Fig. 1E). We did not 
observe significant elevations in pro-inflammatory cytokines interleukin (IL)-6 (Fig. 1E). We observed a direct relationship between serum MCP-1 levels and pro-inflammatory monocytes over the course of infection, while pDCs and neutrophil frequencies were inversely related to I-TAC and IL-8 levels respectively (Fig. 1F). While no statistically significant changes occurred with IL-6 or IL-10, both cytokines were correlated over the course of infection. The brisk and transient innate immune dynamics following exposure to SARS-CoV-2 are consistent with the minimal changes observed in body weight and oxygen saturation levels and mild overall disease pathology.

To assess the increase in CD4 $\mathrm{T}_{\text {fh }}$ cells attributable to SARS-CoV-2, we profiled peripheral blood to capture effector T cell responses. No evidence of general lymphopenia was observed (Figure S1D). Frequency and absolute counts of activated CXCR5 ${ }^{+}$CD $4 \mathrm{~T}_{\text {fh }}$ cells, identified by co-expression of Ki-67 and PD-1, significantly increased in all animals at Day 7 regardless of plasma intervention (Fig. 1G-H). At the apex of the effector response, $\mathrm{Ki}-67^{+} \mathrm{CD} 4 \mathrm{~T}$ cells, specifically the $\mathrm{T}_{\mathrm{h}} 1$ but not the $\mathrm{T}_{\mathrm{fh}}$ subset was strongly associated with proliferating CD8 T cells (Fig. 1I). In turn, we observed strong antigen-dependent induction of CD8 T cells evidenced by the association between SARS-CoV-2 vRNA from nasal washes and proliferating (Ki67+) CD8 T cells.

Evaluation of infection-induced changes in CD4 T cell differentiation at Day 7 revealed a strong phenotypic shift to $T_{h} 1$ effectors (CXCR3+), $T_{h} 1$ polarized $T_{f h}$ cells (CXCR3 $+C X C R 5+$ ) and $T_{h} 1 T_{h} 17$ (CXCR3 + CCR6+) CD4 T cells (Fig. 1 J, Figure S2A). Correspondingly, the data showed accumulation of CD4 $T_{h} 1$ cells at Day 7 (Figure S2B). While $T_{h} 2$ CD 4 cells did not peak at Day 7 , there was an increase of $\mathrm{T}_{\mathrm{h}} 17 \mathrm{CD} 4$ cells (Figure S2C-D). Using the acute activation marker, inducible costimulator (ICOS), to identify proliferating (Ki67+) CD4 T cells at Day 7 (Fig. 1K), we found that ICOS + CXCR5- and CXCR5 + CD4 $T$ cells subsets expressed the $T_{h} 1$ marker signaling lymphocyte adhesion molecule (SLAM) and the effector molecule CX3CR1, a marker potentially for newly generated memory CD4 T cell subsets, consistent with their activation status (Fig. 1L). Neither the ICOS + CXCR5- nor the CXCR5 + CD4 T cell subsets downregulated CD28 and both subsets expressed CCR7 at levels comparable to or greater than naive cells, indicative of a lymph node-homing phenotype. To assess CD4 T cell functionality, cytokine production was evaluated ex vivo following stimulation with PMA and ionomycin. Two distinct CD4 T cells were identified $-a$ degranulating CD107 $a+b$ subset with the majority of degranulating CD4 T cells expressing interferon gamma (IFNY) and TNFa but not IL-2 or IL-17; and an IL-21-producing subset (Figure S2E). In contrast, the majority of IL-21-expressing cells produced IL-2, IL-17 and co-produced TNFa and IFNy. Thus, CD4 T cell polyfunctionality was preserved during SARS-CoV-2 infection. Despite the increase in activated $\mathrm{T}_{\text {fh }}$ cells, levels of CXCL13 did not increase significantly following SARS-CoV-2 infection (Figure S2F).

CD4 $\mathrm{T}_{\text {fh }}$ cells targeting the spike $(\mathrm{S})$ and nucleocapsid $(\mathrm{N})$ proteins are generated following SARS-CoV-2 infection. Based on the significant increase in systemic CD4 $\mathrm{T}_{\text {fh }}$ cells following SARS-CoV-2 exposure, we sought to understand splenic involvement during the germinal center phase of the immune response. To this end, we quantified GC $T_{\text {fh }}$ cells in the spleen at necropsy and compared the values to those seen in 
animals that had not been exposed to SARS-CoV-2. The results suggested the initiation of a GC response within the spleen following infection (Figure S3A). We observed that the majority of the GC $T_{f h}$ cells did not express Foxp3 indicating that $G C T_{\text {fh }}$ cells predominated over the GC T follicular regulatory cell $\left(T_{\text {fr; }}\right.$ CXCR5+, PD-1++, Foxp3+) subset (Figure S3B, Tregs were defined as CD95 + CXCR5- Foxp3+). To conclusively assess SARS-CoV-2-induced responses, we stimulated cryopreserved splenocytes with mega pools - overlapping peptides covering multiple T cell epitopes in $S, N$, and membrane (M) proteins, and spanning the open reading frames (ORF1,3,8) of SARS-CoV-2. PMA/lonomycin was used as a positive control while DMSO-treated cells served as negative controls. Using activation induced marker (AIM) assay, SARS-CoV-2-specific CD4 T cells were identified based on co-expression of OX40 and CD25 (Fig. 2A, Table S2).

Following subtraction of AIM + responses in DMSO-treated cells, CD4 T cell responses to $\mathrm{S}$ and $\mathrm{N}$ were detected. Furthermore, PD-1 + +GC $T_{\text {fh }}$ cells, reactive to $S, N$, and $M$ were observed indicative of SARSCoV-2-induced GC response in the spleen (Fig. 2B). It should be noted, however, that responses to S, N, M were also detected in unexposed animals suggestive of cross-reactive $T$ cells to endemic coronaviruses, as has been reported in humans ${ }^{15} 23$. Evaluation of CD4 T cell polyfunctionality in the spleen by ICS in response to $\mathrm{PMA}$ /lonomycin stimulation revealed that CXCR5 + CD4 T cells were clearly distinguishable from CXCR5- subsets in their ability to co-produce IFNy, IL-2, TNFa, and IL-21. In contrast, the CXCR5subset produced little IL-21 yet was able to co-produce IL-2 and TNFa, or, alternatively, either IFNy, IL-2, or TNFa. In contrast, CD8 T cells were predominantly IFNy producers (Fig. 2C-D). Consistent with data from the spleen, antigen-specific responses against $S$ and $N$ were also observed in peripheral blood at Day 7 (Fig. 2E-F). Together, these data demonstrate that S- and N-specific CD4 $\mathrm{T}_{\text {fh }}$ cells are elicited following SARS-CoV-2 infection.

\section{SARS-CoV-2 infection induces germinal center responses in mediastinal lymph nodes.}

Having established that SARS-CoV-2 stimulates the production of CD4 $\mathrm{T}_{\text {fh }}$ cells, we next assessed whether $T_{h} 1$ effector $C D 4 T$ cells were induced in the lung. Subsequent to collagenase digestion, singlecell suspensions isolated from the lung were stained with a panel of markers to delineate activated CD4 T cells. We evaluated expression of Granzyme B and PD-1, both antigen-induced activation markers; mucosal homing receptors $a_{4} B_{7}, C C R 6$, and the $T_{h} 1$ receptor $C X C R 3$ within CD69 + and CD69- CD 4 T cell subsets (Fig. 3A). The expression pattern of Granzyme B, PD-1, and CXCR3 in lung CD4 T cells was indicative of $a T_{h} 1$ effector $C D 4$ response (Fig. 3B). Furthermore, histopathology of the lung showed development of Bronchus-associated lymphoid tissue (Figure S4A) substantiating induction of adaptive immune responses. Gross examination of the mediastinal lymph nodes was consistent with lymphadenopathy (Figure S4B). Single cell suspensions obtained from lymph nodes were stained with a panel of markers to define GC T $T_{\text {fh }}$ cells, GC B cells, and follicular dendritic cells (FDCs). As illustrated, mediastinal lymph nodes showed a distinct CXCR5 + PD-1 ++GC $T_{\text {fh }}$ subset and Bcl- $6+C D 20+G C$ B cells (Fig. 3C). FDCs were identified based on expression of the complement receptor CD21 (clone B-Ly4; 
Figure S4C), within the CD45- CD3- CD20- cell population ${ }^{24}$. The number of FDCs strongly correlated with the frequencies of both GC B cells and GC $T_{\text {fh }}$ cells (Figure S4D).

Quantifying the expression of canonical GC markers showed that Bcl- 6 was exclusively expressed by GC $B$ cells and to a lesser extent by GC $T_{\text {fh }}$ cells (Fig. 3D). FDC markers CD21 and platelet-derived growth factor receptor b (CD140b) ${ }^{25}$ were also expressed by GC $T_{\text {fh }}$ and $B$ cells (Fig. 3D). An increase in expression of the $T_{h}$ 1-chemokine receptor, $C X C R 3$, on $G C T_{\text {fh }}$ cells was consistent with the phenotype of cells responding to viral infection. While GC B cells displayed heterogeneity in CXCR3 expression, FDCs were uniformly negative for this marker. The increased number of GC $T_{\text {fh }}, G C B$ cells and FDCs (Figure $E$ ) as well as the higher relative expression of CXCR3 in mediastinal lymph nodes compared to cervical and mesenteric lymph nodes indicated an active immune response to viral infection (Fig. 3F). Consistently, we observed SARS-specific responses by GC $T_{\text {fh }}$ cells in the mediastinal lymph node (Fig. 3G, Figure S4E).

\section{Humoral responses to SARS-CoV-2 are dominated by IgG antibodies}

Studies in humans demonstrate that greater than $90 \%$ of SARS-CoV-2 patients develop binding antibodies to $S$ antigen within 10 days of symptom onset ${ }^{26,27}$. However, the kinetics of the early antibody response to $\mathrm{S}$ and $\mathrm{N}$ proteins and the contributing antibody isotypes, specifically in the setting of mild or asymptomatic clinical illness, are not well-defined. Here, we quantified concentrations of serum antibodies to S1, S2, and N antigens, and used a secondary antibody specific for macaque IgG to distinguish de novo IgG antibodies from passively infused human CP IgG antibodies. The data showed $\lg M$ and $\lg G$ seroconversion to $S 1$ and $S 2$ proteins in all animals by day 7 post-infection, with the exception of one animal (Fig. 4A \& B). This is consistent with reports that S- or RBD-specific IgG and IgM antibodies often appear simultaneously in blood of most humans infected with 2002 SARS or CoV-2 $26-$ 28. Antibody responses to the $\mathrm{N}$ protein in humans are reported to increase 10 days following disease onset ${ }^{29,30}$, and interestingly, $\mathrm{N}$-specific IgG was evident in all macaques by day 7 but $\mathrm{N}$-specific IgM was not increased significantly (3-fold over baseline values) until day 10 in most animals. In addition, $50 \%$ of the animals failed to demonstrate a significant IgA response to all SARS CoV-2 proteins within 10 days of infection (Fig. 4C). However, we should note that analysis of some necropsy sera suggested that IgA antibodies continue to increase after day 10 (data not shown).

Evaluation of the magnitude of post-infection antibody responses in animals that did not receive CP plasma clearly indicated that IgG dominated the humoral response to all SARS CoV-2 proteins (Fig. 4D). On day 10, we observed strong correlations between S1-specific IgG and IgM and between N-specific IgA and IgG (Fig. 4E). Also consistent with reports in infected humans, we observed a strong correlation between neutralization antibody titers and concentrations of anti-RBD IgG antibodies on day 10 (Fig. 4E). Together, these data show rapid development of binding and neutralizing antibodies following SARS-CoV2 infection in the context of mild or absent clinical symptoms. The appearance of antiviral IgG antibodies 
by day 7 with delayed induction of IgA responses suggests that early class-switching occurs after SARSCoV-2 infection and is likely promoted by $T_{h} 1$-type $T_{\text {fh }}$ cells.

\section{Discussion}

The importance of CD4 $\mathrm{T}_{\text {fh }}$ cells in the generation of plasma cells, critical for persistent antibody, places a premium on understanding the $\mathrm{T}_{\mathrm{fh}}$ and GC response following SARS-CoV-2 infection. The present study adds to our understanding of immune responses to SARS-CoV-2 in three significant ways. First, we demonstrate that robust $T_{h} 1-T_{f h}$ responses are observed following SARS-CoV-2 infection. Second, $T_{f h}$ responses focused on $\mathrm{S}$ and $\mathrm{N}$ are seen within lymph nodes, circulate through peripheral blood, potentially seeding the spleen. Lastly, we show that acute antibody kinetics are characterized by induction of IgG, predominantly to S1, indicative of early class switching. Taken together, these data demonstrate that productive $T_{\text {fh }}$ responses are elicited following SARS-CoV-2 infection in healthy adult rhesus macaques. While the $T_{h} 1$-bias of $T_{\text {fh }}$ cells following infection is expected due to the robust interferon response following SARS-CoV-2, this skewing is important to note, as weak interferon responses observed in COVID patients could hamper shoring up effective antiviral antibody and CD8 T cell responses ${ }^{31}$.

Several studies have examined the kinetics of antibody responses in humans after the onset of symptoms and three unifying themes emerge from these data. First, in the majority of patients, antibodies to RBD of the $\mathrm{S} 1$ subunit are induced between 8-10 days of symptom onset, and levels of these antibodies correlate strongly with neutralizing titers ${ }^{26,27,32}$. Second, plasma from the majority of COVID-19 convalescent patients does not contain high levels of neutralizing activity ${ }^{33}$. Third, plasma antibodies in infected individuals that do develop neutralizing antibodies are minimally mutated ${ }^{34}$. These data suggest that CD8 T cells may contribute to the control of SARS-CoV-2, and while a protracted germinal center response may not be critical for the generation of neutralizing antibodies it could improve antibody durability by enhancing plasma cell numbers. Our data add to this developing narrative by showing that in the setting of mild/asymptomatic illness, antibody responses are generated and characterized by the predominance of IgG. Intriguingly, we observed that IgM and IgG antibodies to the $\mathrm{S} 1, \mathrm{~S} 2$ and N proteins were produced concurrently.

While it remains unknown whether immune responses elicited when naturally infected by SARS-CoV-2 will protect from re-infection, studies in rhesus macaques show that infection does protect against rechallenge, 28-35 days post first infection, signifying that some degree of a protective immune response follows infection ${ }^{21}$. In this context, the finding that infection induces CD4 helper responses targeting major structural proteins on the virus suggests that infection is capable of producing effective CD4 help for CD8 T cells and antibody responses. Indeed, antibodies against the RBD region in S1 are elicited in the vast majority of COVID-19 patients along with robust CD4 T cell responses ${ }^{27}$. Our data show that spike epitopes are immunogenic to both $T$ and $B$ cells and suggests that induction of these responses by vaccines may confer protection. While our discovery of $\mathrm{N}$ - and S-specific CD4 T cells in the spleen is 
intriguing at present we are unable to distinguish whether these cells represent cells that are seeded from circulation or are elicited de novo via trafficked antigen. Further studies are needed to tease apart the possibilities as this is central to understanding determinants of protective immunity. In sum, the data suggest that vaccine platforms inducing $T_{h} 1 C D 4$ helper and $T_{\text {fh }}$ helper responses are likely to succeed in eliciting robust CD8 T cell and antibody responses against SARS-CoV-2. Similar observations of circulating $T_{\text {fh }}$ cells in vaccinated and infected humans support this hypothesis ${ }^{14,35,36}$.

In summary, the current study adds to our understanding of the CD4 helper responses to SARS-CoV-2 infection and provides an important foundation for harnessing the mechanisms that stimulate robust CD4 $\mathrm{T}_{\mathrm{fh}}$ responses in the context of an effective vaccine.

\section{Methods}

Rhesus Macaques. Eight colony-bred Indian origin rhesus macaques (Macaca mulatta) were housed at the California National Primate Research Center and maintained in accordance with American Association for Accreditation of Laboratory Animal Care guidelines and Animal Welfare Act/Guide. As described by us ${ }^{37}$, strict social distancing measures were implemented at the CNPRC at the start of the pandemic in March to reduce risk of human-to-rhesus SARS-CoV-2 transmission. Animals were screened for SARS-CoV-2 and housed in barrier rooms with increased PPE requirements prior to study assignment. Animals were four to five years of age with a median weight of $8.6 \mathrm{~kg}$ (range 5.4-10.7 kg), were SIV- STLVSRV-. Animals were seronegative for SARS-CoV-2 at study initiation. Sex distribution within experimental groups was as follows; Infected ( $\mathrm{n}=3$ females, $\mathrm{n}=1$ male); Infected + Convalescent Plasma ( $\mathrm{n}=2$ males); Infected + Normal Plasma ( $\mathrm{n}=2$ males). Table S1 provides details of the animals in the study. For blood collection, animals were anesthetized with $10 \mathrm{mg} / \mathrm{kg}$ ketamine hydrochloride injected i.m. For virus inoculation and nasal secretion sample collection, animals were additionally anesthetized with 15-30 $\mathrm{ug} / \mathrm{kg}$ dexmedetomidine $\mathrm{HCl}$ inject i.m. and anesthesia was reversed with $0.07-0.15 \mathrm{mg} / \mathrm{kg}$ atipamezole $\mathrm{HCl}$ injected i.m.

Virus and inoculations. SARS-CoV-2 virus was isolated from the nasal swab of a COVID-19 patient with acute respiratory distress syndrome admitted to University of California, Davis Medical Center, Sacramento ${ }^{38}$. Vero cells (ATCC CCL-81) were used for viral isolation and stock expansion. The passage 2 viral stock (SARS-CoV-2/human/USA/CA-CZB-59X002/2020) used for animal inoculations had a titer of $1.2 \times 10^{6} \mathrm{PFU} / \mathrm{ml}$ (corresponding to $2 \times 10^{9} \mathrm{VRNA}$ ) (Genbank accession number: MT394528). To recapitulate relevant transmission routes of SARS-CoV-2, animals were inoculated with $1 \mathrm{ml}$ stock instilled into the trachea, $1 \mathrm{ml}$ dripped intranasally, and a drop of virus stock in each conjunctiva.

Convalescent plasma and infusions. Convalescent plasma was sourced from Vitalant and represented a pool from up to four donors. Plasma was pooled prior to infusion into monkeys. Pooled plasma had a $\mathrm{nAb}$ titer of 1:1149, binding antibody titers for SARS-CoV-2 antigen were as follows; anti-S1-lgG, 24.5 $\mu \mathrm{g} / \mathrm{ml}$; anti-S2 lgG, $2.9 \mu \mathrm{g} / \mathrm{ml}$; anti-NC IgG, $10.7 \mu \mathrm{g} / \mathrm{ml}$. Normal plasma was collected prior to the COVID19 pandemic and was negative for SARS-Cov2 antibody. Concentrations were as follows; anti-S1-IgG, 
$0.00004 \mu \mathrm{g} / \mathrm{ml}$; anti-S2 lgG, $0.003 \mu \mathrm{g} / \mathrm{ml}$; anti-N IgG, $0.001 \mu \mathrm{g} / \mathrm{ml}$. Twenty fours following virus inoculation, animals were infused with plasma at $4 \mathrm{ml} / \mathrm{kg}$ volume (total volume infused was 33-39 $\mathrm{ml}$ ) at an infusion rate of $1 \mathrm{ml} /$ minute. Control animals $(n=4)$ were not infused.

Specimen collection and processing. On days $2,4,5,7,8$, and 10, a 5 -french feeding tube was inserted into the nasal cavity. $2 \mathrm{ml}$ PBS were instilled through each nostril and the maximum volume was aspirated, secretions were spun at $800 \mathrm{~g}$ for $10 \mathrm{~min}$ and $1 \mathrm{ml}$ of supernatant and cell pellet were lysed in $3 \mathrm{ml}$ Trizol LS for RNA isolation. EDTA-anticoagulated blood was collected on Days $0,2,4,7$, and 10 for immunophenotyping. PBMCs were isolated from whole blood collected in CPT vacutainer tubes, sampled at Day 7 and necropsy, by density gradient centrifugation as previously described ${ }^{39}$. For serum, coagulated blood was centrifuged at $800 \mathrm{~g}$ for 10 min to pellet clotted cells, followed by extraction of supernatant and storage at $-80^{\circ} \mathrm{C}$. Lymph nodes, spleen, and lung tissue were obtained at necropsy and digested enzymatically using collagenase followed by manual disruption to obtain single cell suspensions for flow cytometry based assays.

Activation induced Marker (AIM) assay. Cells were stimulated with overlapping peptide pools representing SARS-CoV-2 and responding cells were identified by upregulation of activation markers, as described previously ${ }^{39,40}$. All antigens were used at a final concentration of $2 \mathrm{mg} / \mathrm{mL}$ in a stimulation cocktail made with using $0.2 \mathrm{mg}$ of CD28 and $0.2 \mathrm{mg}$ CD $49 \mathrm{~d}$ costimulatory antibodies per test. Unstimulated controls were treated with volume-controlled DMSO (Sigma-Aldrich). Tubes were incubated in $5 \% \mathrm{CO}_{2}$ at $37^{\circ} \mathrm{C}$ overnight. Following an $18 \mathrm{~h}$ stimulation, the cells were stained, fixed, and acquired the same day. AIM assays on splenocytes and mediastinal lymph nodes were performed on cryopreserved cells (Table S2). AIM assay on day 7 PBMCs were performed on fresh cells. Phenotype panel on LNs and PBMCs was performed using standard flow cytometry assays.

\section{vRNA quantitation by quantitative real time polymerase chain reaction (qRT-PCR)}

Trizol lysed nasal samples were processed using a modified Qiagen RNeasy Lipid Tissue Mini Kit protocol. Briefly, $5.6 u$ polyacryl carrier was added to trizol lysate, followed by $1 / 10$ volume BCP and phase separated as described in Qiagen protocol. 8ul of eluted RNA was DNase treated with ezDNase per kit instructions and converted to CDNA with Superscript IV using random primers in a 20ul reaction and quantified in quadruplicate by qPCR on an Applied Biosystems QuantStudio 12K Flex Real-Time PCR System using Qiagen QuantiTect Probe PCR Mastermix with primers and probe that target the SARS-CoV2 Nucleocapsid (forward 5'-GTTTGGTGGACCCTCAGATT-3', reverse 5'-GGTGAACCAAGACGCAGTAT-3', probe 5'-/5-FAM/TAACCAGAA/ZEN/TGGAGAACGCAGTGGG/3IABkFQ/-3').

Serum cytokines. Serum cytokines. Luminex ${ }^{\circledR}$ (NHP Cytokine Luminex Performance Pre-mixed kit, R\&D, FCSTM21) was performed to evaluate cytokines in rhesus macaque sera. The assay was performed according to the manufacturer's protocol. The beads for each sample, control, and standard curve point were interrogated in a Luminex ${ }^{\circledR} 200$ dual laser instrument (Luminex, Austin, TX) which monitors the spectral properties of the beads and amount of associated phycoerythrin (PE) fluorescence for each 
sample. XPONENT ${ }^{\circledR}$ software was used to calculate the median fluorescent index and calculate the concentration for each cytokine in

Flow cytometry. Cell staining was performed as previously described. Whole blood and single cell suspensions from the lung and lymph nodes were stained fresh and acquired the same day. Staining on spleen was performed on cryopreserved samples. Fluorescence was measured using a BD Biosciences FACSymphony $^{\mathrm{TM}}$ with FACSDiva ${ }^{\mathrm{TM}}$ version 8.0.1 software. Compensation, gating and analysis were performed using FlowJo (Version 10). Reagents used for flow cytometry are listed in Table S2.

BAMA for IgG and IgM antibodies to S1, S2 and N proteins A customized BAMA was developed to simultaneously measure antibodies to the following recombinant SARS CoV-2 proteins (all from SinoBiologicals, Wayne, PA): S1 (\#40591-V08H), S2 extracellular domain (\#40590-V08B) and nucleocapsid (N; \#40588-V08B). Briefly, proteins were dialyzed in PBS and conjugated to Bioplex Pro carboxylated magnetic beads (BioRad, Hercules, CA) as previously described ${ }^{39}$. Standard and serum samples treated with $1 \%$ TritonX-100 detergent were serially diluted in PBS containing $1 \%$ BSA, $0.05 \%$ azide, and $0.05 \%$ Tween-20 and mixed with beads overnight at $1100 \mathrm{rpm}$ and $4^{\circ} \mathrm{C}$. The following day, the beads were washed and treated with biotinylated antibody followed by neutralite avidin-phycoerythrin (Southern Biotechnology Associates: SBA, Birmingham, AL) as described (Phillips 2017). A BioRad Bioplex 200 and BioManager software were used to measure fluorescent intensity and construct standard curves for interpolation of antibody concentrations in test samples.

The standard was pooled serum from macaques infected for 11-14 days with SARS CoV-2. The following humanized ( $\lg \mathrm{G} 1$ ) monoclonal antibodies were used to estimate concentrations of $\lg$, IgM, and IgA antibodies in the rhesus serum standard: anti-S1 RBD (Genscript \#HC2001), anti-S2 (SinoBiologicals \#40590-D001) and anti-NC (Genscript \#HC2003). Human and rhesus IgG antibodies were both detected in these calibration assays using biotinylated affinity-purified goat anti-human IgG $Y$ chain polyclonal antibody (SBA \#2048-08). In subsequent BAMA assays for SARS CoV-2-specific rhesus macaque IgG antibodies, biotinylated mouse anti-monkey IgG $y$ chain monoclonal antibody (SBA cat\#4700-08) was used as the secondary antibody. IgM antibodies were detected using biotinylated affinity-purified goat anti-human IgM $\mu$ chain polyclonal antibody (SBA\#2020-08) which cross-reacts well with macaque IgM. Results obtained for IgM in the rhesus standard were multiplied by 3.3 to account for under-estimation by the monomeric IgG monoclonal antibody standard. Macaque IgA antibodies were detected with the antibodies described below. IgG, IgM or IgA antibodies had to be increased 3-fold over the day 0 value to be considered significant

\section{ELISA for SARS-specific IgA and antibodies to RBD}

These assays were done using methods similar to those described ${ }^{41}$ and Immulon 4 microtiter plates (VWR, Radnor, PA) coated with 100ng per well of S1, S2, N or RBD protein (SinoBiological \#40592-VNAH). For IgA assays, a pooled rhesus serum collected day 14 after infection with SARS CoV-2 was used as standard after depletion of IgG using GE Healthcare Protein G Sepharose (Sigma, St. Louis, MO) as 
described ${ }^{41}$. Test sera were also depleted of IgG to facilitate detection of low levels of IgA antibodies. Macaque IgA was detected using a mixture of biotinylated clone 10F12 (NHP Reagent Resource) and biotinylated clone 40.15.5 (Ward et al 1995) anti-rhesus IgA monoclonal antibodies, which do not crossreact with human IgA and, when combined, appear to recognize all allotypes of rhesus macaque IgA (Kozlowski, personal observation). For RBD IgG assays, the above pooled rhesus serum standard and secondary monoclonal antibody specific for macaque lgG were used.

Neutralizing assay Pseudovirus neutralization assay was performed as described ${ }^{42}$.

Statistics Statistical analyses were performed using GraphPad Prism 8.4.2. Within group comparisons, such as immune responses and antibody levels at different time points, were done using the two-tailed Wilcoxon matched-pairs signed rank test. For correlation analysis, the two-tailed Spearman rank correlation test was used.

Materials Availability. The study generated SARS-CoV-2/human/USA/CA-CZB-59X002/2020 and is available upon request from CJM (Genbank accession number: MT394528).

\section{Declarations}

\section{AUTHOR CONTRIBUTIONS}

DHOC, KKVR, CJM, SSI supervised the project. SRE, JWR, TDC, SJM, JHM, DHOC, KVR, CJM, SSI conceptualized study design. SRE, YSL, JWR, BAS, NKN, SSI performed flow assays. SRE, YSL, JWR, SSI analyzed and interpreted flow data. RLS performed clinical assessments. TDC, JDD grew up virus stock and processed nasal washes. JD performed vRNA analyses using qRT PCR. JRR, KLO, ZMM performed histopathological analysis. JW, JU, RI, JLY conducted assays and assisted with necropsy. DW, AS, MS, NKT, GS, MPB provided key reagents. PAK, KDW and JCS performed antibody assays and SF performed the pseudovirus neutralization assays. SSI wrote the original draft of the manuscript. All authors reviewed and edited the manuscript.

\section{ACKNOWLEDGEMENTS}

The authors are grateful to Lourdes Adamson and Nicole Drazenovich for BSL3 training and facilitating access to CIID BSL3. The authors are grateful to Greg Hodges for facilitating the animal experiments in CNPRC ABSL3. We are extremely grateful to the primate center staff Wilhelm Von Morgenland, Miles Christensen, David Bennet, Vanessa Bakula, James Schulte, Jose Montoya, Joshua Holbrook, John McAnelly, Christopher Nelson, and David Bennett for animal sampling in ABSL3 at the CNPRC. Mark Allen provided necropsy technical expertise in the BSL-3 necropsy suite. The authors are grateful to Amanda Carpenter, Peter Nham, and Bryson Halley at the Primate Assay Laboratory Core. We thank Larry Dumont at VIR for the CP and Linda Fritts for assistance with PCR assays. We are grateful to David Asmuth for 
facilitating IRB approval to procure clinical samples. We thank Jeffrey Roberts, Lisa Miller, Marcelo Kuroda and the CNPRC leadership for their support in facilitating these experiments and acknowledge assay resources provided by the Primate Assay Laboratory Core and CNPRC base grant. The 10F12 antimonkey IgA monoclonal antibody was obtained from the NIH Nonhuman Primate Reagent Resource supported by Al126683 and OD010976. This work was supported by internal seed grants to CNRPC and CIID, R21 Al143454-02S1 (SSI), FAST GRANT- George Mason University (SSI), NIH Contract \# 75N9301900065 (A.S. and D.W), and the CNPRC base grant P510D011107

\section{DECLARATION OF INTERESTS}

A.S. is listed as inventors on a provisional patent application covering findings reported in this manuscript. A.S. is a consultant for Gritstone, Flow Pharma, and Avalia. The remaining authors have declared that no conflict of interest exists.

Further information and requests for resources and reagents should be directed to and will be fulfilled by the Lead Contact, Smita S. lyer (smiyer@ucdavis.edu).

\section{ANIMAL ETHICS}

Rhesus Macaques. Eight colony-bred Indian origin rhesus macaques (Macaca mulatta) were housed at the California National Primate Research Center and maintained in accordance with American Association for Accreditation of Laboratory Animal Care guidelines and Animal Welfare Act/Guide. All studies were approved by the University of California Davis Institutional Animal Care and Use Committee (IACUC).

\section{References}

1. WHO announces COVID-19 outbreak a pandemic. World Health Organization (2020). (2020).

2. Huang, C. et al. Clinical features of patients infected with 2019 novel coronavirus in Wuhan, China. Lancet 395, 497-506, doi:10.1016/S0140-6736(20)30183-5 (2020).

3. Corey, L., Mascola, J. R., Fauci, A. S. \& Collins, F. S. A strategic approach to COVID-19 vaccine R\&D. Science 368, 948-950, doi:10.1126/science.abc5312 (2020).

4. Graham, B. S. Rapid COVID-19 vaccine development. Science 368, 945-946, doi:10.1126/science.abb8923 (2020).

5. Amanat, F. \& Krammer, F. SARS-CoV-2 Vaccines: Status Report. Immunity 52, 583-589, doi:10.1016/j.immuni.2020.03.007 (2020).

6. Pulendran, B. \& Ahmed, R. Immunological mechanisms of vaccination. Nat Immuno/ 12, 509-517, doi:10.1038/ni.2039 (2011).

7. Amanna, I. J., Carlson, N. E. \& Slifka, M. K. Duration of humoral immunity to common viral and vaccine antigens. N Engl J Med 357, 1903-1915, doi:10.1056/NEJMoa066092 (2007). 
8. Renhong Yan, Y. Z., Yaning Li3, Lu Xia, Yingying Guo1,2, Qiang Zhou. Structural basis for the recognition of the SARS-CoV-2 by full-length human ACE2. Science, doi:Structural basis for the recognition of the SARS-CoV-2 by full-length human ACE2 (2020).

9. Bentebibel, S. E. et al. ICOS(+)PD-1(+)CXCR3(+) T follicular helper cells contribute to the generation of high-avidity antibodies following influenza vaccination. Sci Rep 6, 26494, doi:10.1038/srep26494 (2016).

10. Crotty, S. T Follicular Helper Cell Biology: A Decade of Discovery and Diseases. Immunity 50, 11321148, doi:10.1016/j.immuni.2019.04.011 (2019).

11. Iyer, S. S. et al. Codelivery of Envelope Protein in Alum with MVA Vaccine Induces CXCR3-Biased CXCR5 + and CXCR5- CD4 T Cell Responses in Rhesus Macaques. J Immuno/ 195, 994-1005, doi:10.4049/jimmunol.1500083 (2015).

12. Thevarajan, I. et al. Breadth of concomitant immune responses prior to patient recovery: a case report of non-severe COVID-19. Nat Med 26, 453-455, doi:10.1038/s41591-020-0819-2 (2020).

13. Mathew, D. et al. Deep immune profiling of COVID-19 patients reveals distinct immunotypes with therapeutic implications. Science, doi:10.1126/science.abc8511 (2020).

14. Juno, J. A. et al. Humoral and circulating follicular helper $\mathrm{T}$ cell responses in recovered patients with COVID-19. Nat Med, doi:10.1038/s41591-020-0995-0 (2020).

15. Grifoni, A. et al. Targets of T Cell Responses to SARS-CoV-2 Coronavirus in Humans with COVID-19 Disease and Unexposed Individuals. Cell, doi:10.1016/j.cell.2020.05.015 (2020).

16. Sekine, T. et al. Robust T cell immunity in convalescent individuals with asymptomatic or mild COVID-19. bioRxiv, 2020.2006.2029.174888, doi:10.1101/2020.06.29.174888 (2020).

17. Wang, Y. D. et al. T-cell epitopes in severe acute respiratory syndrome (SARS) coronavirus spike protein elicit a specific T-cell immune response in patients who recover from SARS. J Virol 78, 56125618, doi:10.1128/JVI.78.11.5612-5618.2004 (2004).

18. Li, C. K. et al. T cell responses to whole SARS coronavirus in humans. J Immunol 181, 5490-5500, doi:10.4049/jimmunol.181.8.5490 (2008).

19. Zhao, J. et al. Airway Memory CD4(+) T Cells Mediate Protective Immunity against Emerging Respiratory Coronaviruses. Immunity 44, 1379-1391, doi:10.1016/j.immuni.2016.05.006 (2016).

20. 10.1101/2020.03.13.990226

Bao, L. et al. Reinfection could not occur in SARS-CoV-2 infected rhesus macaques. bioRxiv, 2020.2003.2013.990226, doi:10.1101/2020.03.13.990226 (2020).

21. Chandrashekar, A. et al. SARS-CoV-2 infection protects against rechallenge in rhesus macaques. Science, doi:10.1126/science.abc4776 (2020).

22. $10.1101 / 2020.06 .05 .136481$

Singh, D. K. et al. SARS-CoV-2 infection leads to acute infection with dynamic cellular and inflammatory flux in the lung that varies across nonhuman primate species. bioRxiv, 2020.2006.2005.136481, doi:10.1101/2020.06.05.136481 (2020). 
23. Braun, J. et al. SARS-CoV-2-reactive T cells in healthy donors and patients with COVID-19. Nature, doi:10.1038/s41586-020-2598-9 (2020).

24. Tew, J. G., Wu, J., Fakher, M., Szakal, A. K. \& Qin, D. Follicular dendritic cells: beyond the necessity of T-cell help. Trends Immuno/ 22, 361-367, doi:10.1016/s1471-4906(01)01942-1 (2001).

25. Krautler, N. J. et al. Follicular dendritic cells emerge from ubiquitous perivascular precursors. Cell 150, 194-206, doi:10.1016/j.cell.2012.05.032 (2012).

26. Long, Q. X. et al. Antibody responses to SARS-CoV-2 in patients with COVID-19. Nat Med 26, 845848, doi:10.1038/s41591-020-0897-1 (2020).

27. Premkumar, L. et al. The receptor binding domain of the viral spike protein is an immunodominant and highly specific target of antibodies in SARS-CoV-2 patients. Sci Immuno/ $\mathbf{5}$, doi:10.1126/sciimmunol.abc8413 (2020).

28. 2005.2003.20084442

Suthar, M. S. et al. Rapid generation of neutralizing antibody responses in COVID-19 patients. medRxiv, 2020.2005.2003.20084442, doi:10.1101/2020.05.03.20084442 (2020).

29. Liu, W. et al. Evaluation of Nucleocapsid and Spike Protein-Based Enzyme-Linked Immunosorbent Assays for Detecting Antibodies against SARS-CoV-2. J Clin Microbio/ 58, doi:10.1128/JCM.0046120 (2020).

30. $10.1101 / 2020.04 .20 .20071423$

Burbelo, P. D. et al. Detection of Nucleocapsid Antibody to SARS-CoV-2 is More Sensitive than Antibody to Spike Protein in COVID-19 Patients. medRxiv, 2020.2004.2020.20071423, doi:10.1101/2020.04.20.20071423 (2020).

31. O'Brien, T. R. et al. Weak Induction of Interferon Expression by SARS-CoV-2 Supports Clinical Trials of Interferon Lambda to Treat Early COVID-19. Clin Infect Dis, doi:10.1093/cid/ciaa453 (2020).

32. Lv, H. et al. Cross-reactive antibody response between SARS-CoV-2 and SARS-CoV infections. bioRxiv, doi:10.1101/2020.03.15.993097 (2020).

33. Robbiani, D. F. et al. Convergent antibody responses to SARS-CoV-2 in convalescent individuals. Nature, doi:10.1038/s41586-020-2456-9 (2020).

34. Seydoux, E. et al. Analysis of a SARS-CoV-2-Infected Individual Reveals Development of Potent Neutralizing Antibodies with Limited Somatic Mutation. Immunity, doi:10.1016/j.immuni.2020.06.001 (2020).

35. Jackson, L. A. et al. An mRNA Vaccine against SARS-CoV-2 - Preliminary Report. N Engl J Med, doi:10.1056/NEJMoa2022483 (2020).

36. Folegatti, P. M. et al. Safety and immunogenicity of the ChAdOx $1 \mathrm{nCoV}-19$ vaccine against SARSCoV-2: a preliminary report of a phase $1 / 2$, single-blind, randomised controlled trial. Lancet, doi:10.1016/S0140-6736(20)31604-4 (2020).

37. $10.1111 /$ jmp. 12483

Yee, J. L. et al. SARS-CoV-2 surveillance for a non-human primate breeding research facility. Journal of Medical Primatology n/a, doi:10.1111/jmp.12483 (2020). 
38. Sanville, B. et al. A Community Transmitted Case of Severe Acute Respiratory Distress Syndrome due to SARS CoV2 in the United States. Clin Infect Dis, doi:10.1093/cid/ciaa347 (2020).

39. Verma, A. et al. Impact of Th1 CD4 Follicular Helper T Cell Skewing on Antibody Responses to an HIV-1 Vaccine in Rhesus Macaques. J Viro/ 94, doi:10.1128/JVI.01737-19 (2020).

40. Havenar-Daughton, C. et al. Cytokine-Independent Detection of Antigen-Specific Germinal Center T Follicular Helper Cells in Immunized Nonhuman Primates Using a Live Cell Activation-Induced Marker Technique. J Immunol 197, 994-1002, doi:10.4049/jimmunol.1600320 (2016).

41. Kozlowski, P. A. et al. Modified wick method using Weck-Cel sponges for collection of human rectal secretions and analysis of mucosal HIV antibody. J Acquir Immune Defic Syndr 24, 297-309, doi:10.1097/00126334-200008010-00001 (2000).

42. Ng, D. et al. SARS-CoV-2 seroprevalence and neutralizing activity in donor and patient blood from the San Francisco Bay Area. medRxiv, 2020.2005.2019.20107482, doi:10.1101/2020.05.19.20107482 (2020).

\section{Figures}




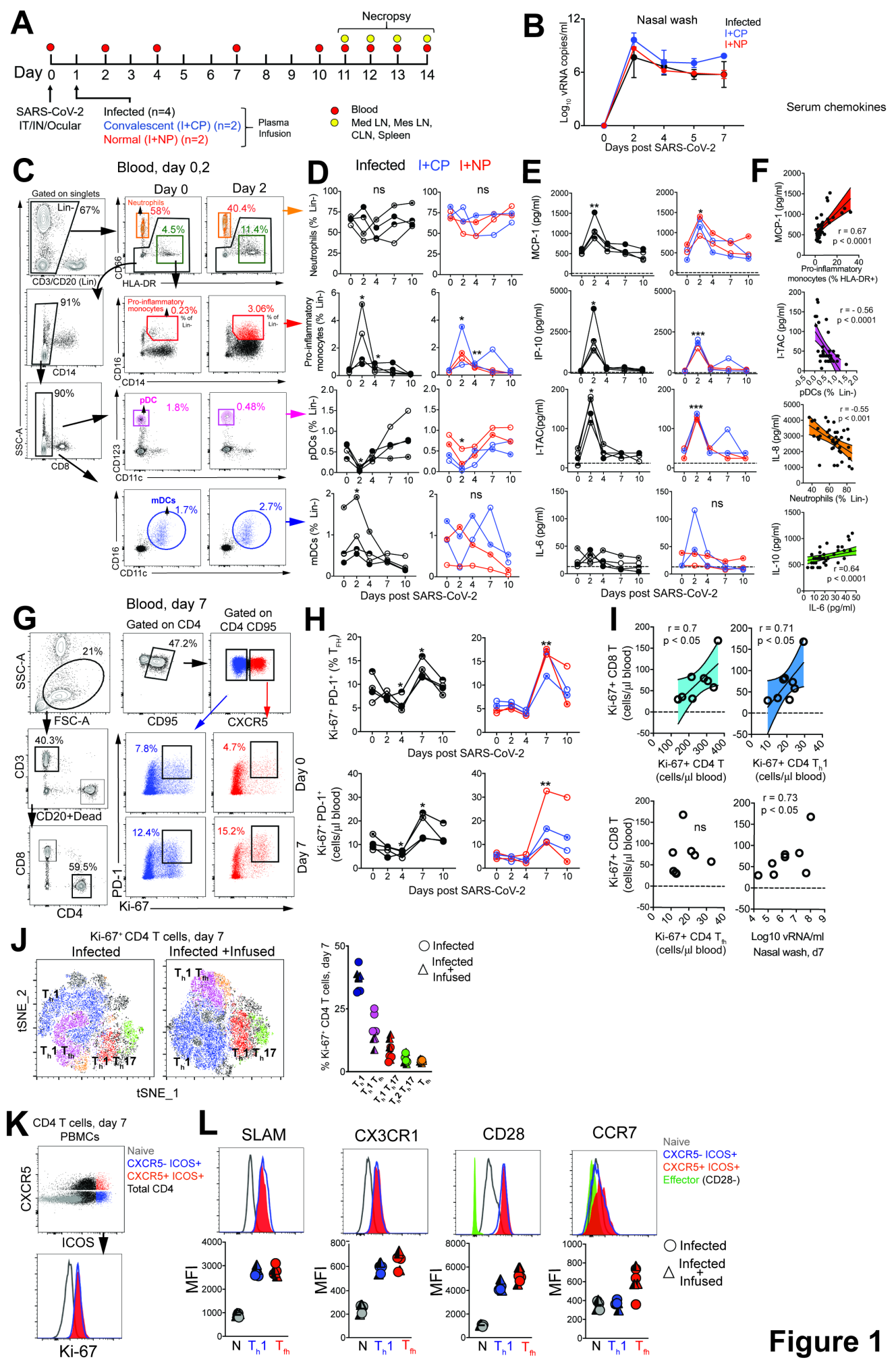

Figure 1

SARS-CoV-2 infection leads to a rapid and transient shift in innate immune responses and increases the number CD4 T follicular helper cells in peripheral blood. (A) Rhesus macaques inoculated with SARS-CoV2 were infused with COVID-19 convalescent plasma (I+CP), or normal plasma (I+NP) or did not receive plasma (infected). (B) Mean viral RNA (+range) in nasal washes (C) Gating strategy for innate immune subsets in blood. (D) Kinetics of innate immune responses ( ${ }^{*} p<0.05,{ }^{* *} p<0.01$ relative to Day 0$)(E)$ 
Serum chemokines MCP-1, IP-10, and I-TAC ( ${ }^{*} p<0.05$, ${ }^{\star \star} p<0.01$, ${ }^{\star \star *} p<0.001$ relative to Day 0$)$. (F) Correlation of innate immune cells against chemokines, and IL-10 vs IL-6. (G) Gating strategy to capture Ki-67+ PD-1+ CD 4 T cells in blood. (H) Kinetics show frequency and absolute counts of Ki-67+ PD-1+ CD4 Tfh cells $\left({ }^{*} \mathrm{p}<0.05,{ }^{*} \mathrm{p}<0.01\right.$ relative to Day 0$)$ (I) correlation plots of Ki-67+CD8 T cells against Ki-67+ CD4 subsets, and vRNA (all day 7) (J) tSNE plot of CD4 Ki-67+ events at Day 7 from infected $(16,197$ events) and infected + infused animals (22,406 events); dot plot shows frequency of Ki-67+ CD4 T cell subsets. (K-L) Histograms and median fluorescence intensity (MFI) dot plots illustrate relative expression of SLAM, CX3CR1, CD28, and CCR7 within four different populations identified at Day 7. Unique symbols identify animals in each of the experimental groups. 

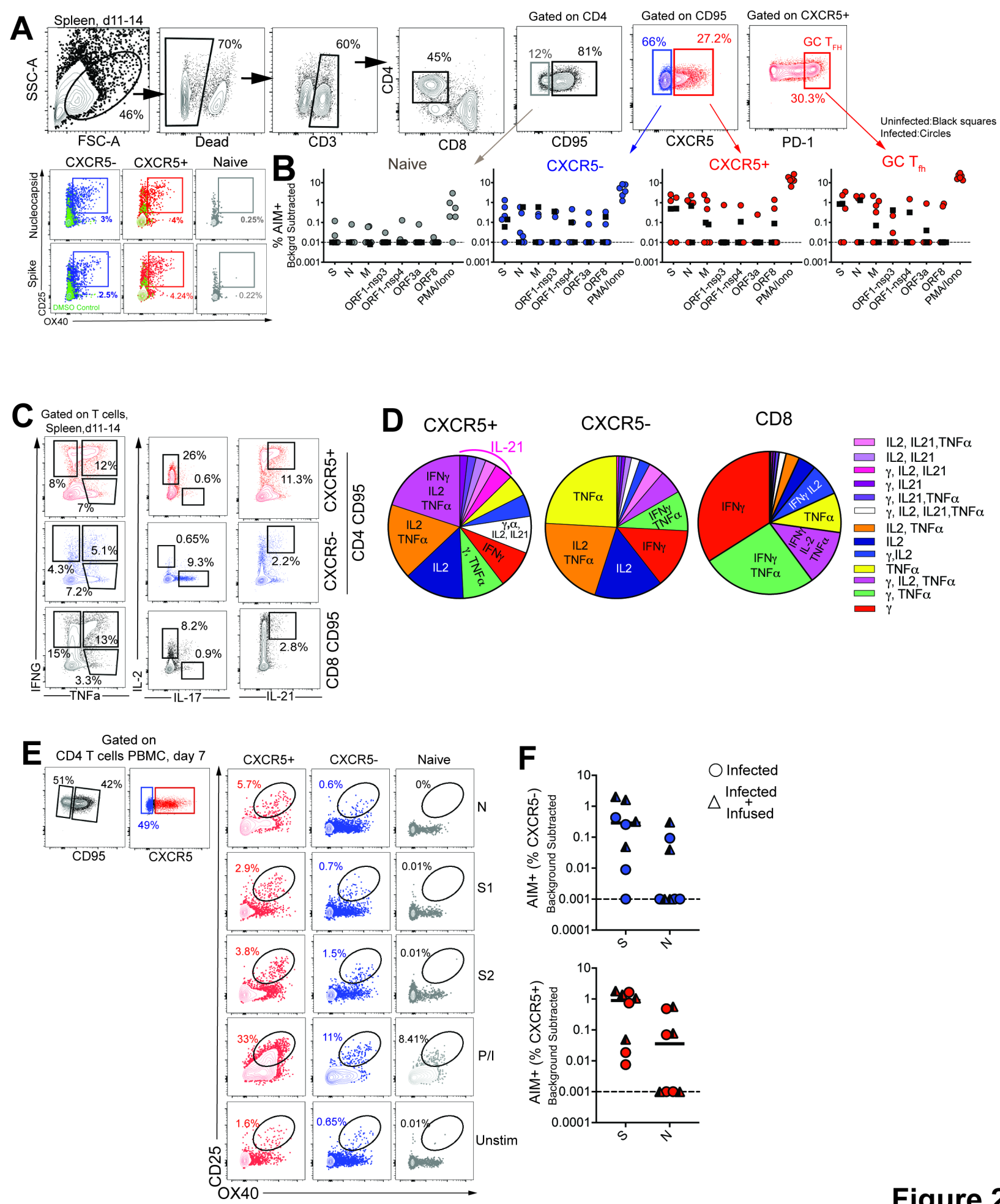

Figure 2

Figure 2

CD4 Tfh cells targeting the spike $(\mathrm{S})$ and nucleocapsid $(\mathrm{N})$ are generated following SARS-CoV-2 infection (A) Gating to identify SARS-CoV-2 specific CD4 T cells following stimulation with peptide megapools (B) Scatter plot showing AIM+CD4 subsets. Dashed line represents undetectable responses assigned a value of $0.01 \%$ (C) Cytokine profiles (IFNy, IL-2, TNF区, IL-17, IL-21) of CXCR5+, CXCR5-, and CD8+CD95+ T cells) in spleen following P/I stimulation. (D) Pie chart shows T cell polyfunctionality. (E) Gating to identify 
SARS-CoV-2 specific CD4 T cells in PBMCs. (F) AIM+ CXCR5- and CXCR5+ CD4 subsets in PBMCs at Day 7. Black squares denote SARS-CoV-2 unexposed animals.
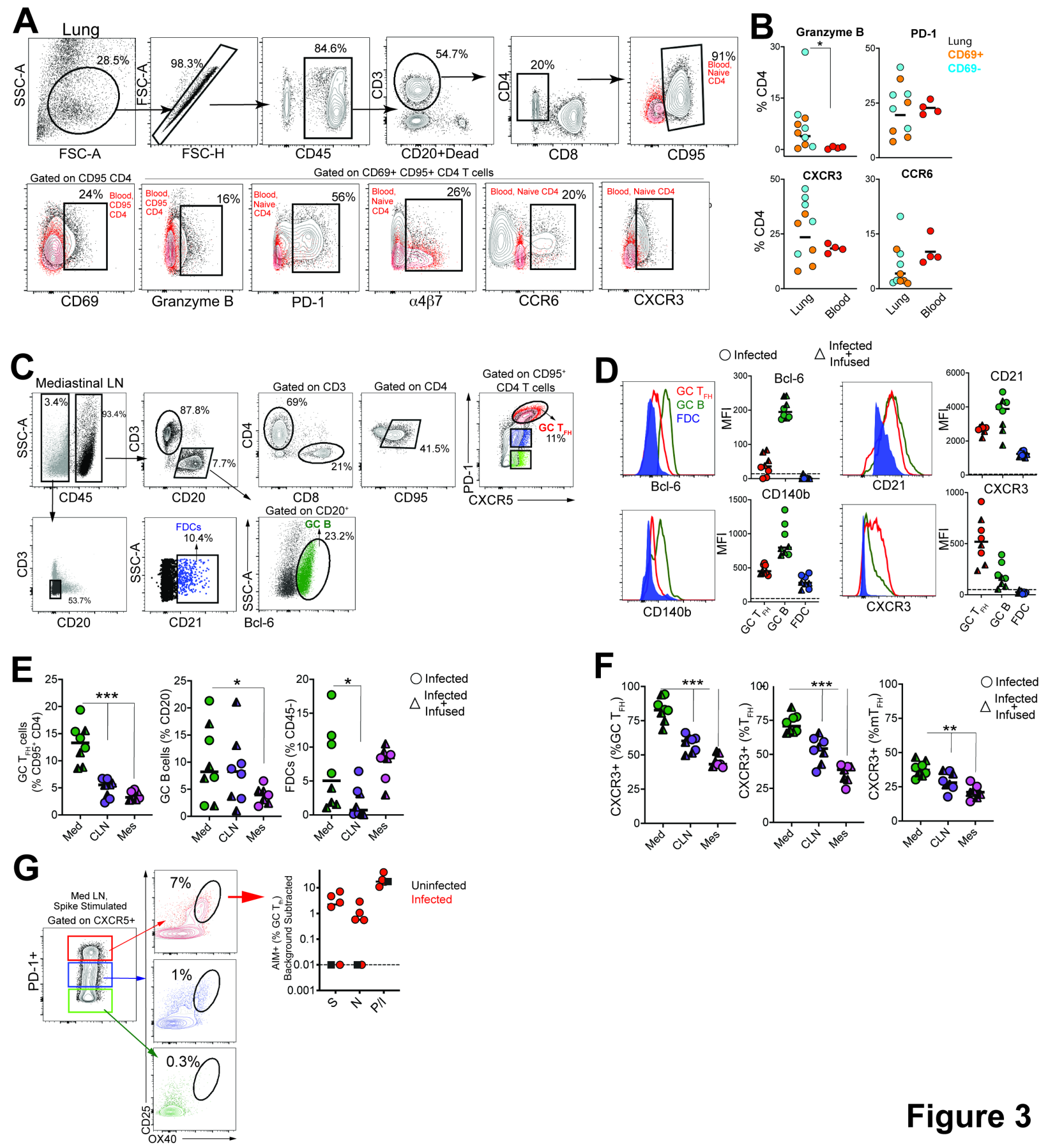

Figure 3

Figure 3

SARS-CoV-2 infection induces germinal center responses in mediastinal lymph nodes. (A) Gating to identify CD4 T cells in lung (B) Dot plots show Granzyme B, PD-1, CXCR3, CCR6 expression on CD69- and CD69+ subsets in lung and CD95+ CD4 T cells in blood ( $n=5)$. (C) Gating for GC Tfh cells, GC B cells, and 
FDCs. (D) Expression of Bcl-6, CD21, CD140b, and CXCR3. (E) Frequency of GC Tfh cells, GC B cells, FDCs significantly higher in mediastinal lymph node ( $\left.{ }^{*} p<0.05, * \star * p<0.001\right)$. (F) Majority of GC Tfh cells in mediastinal lymph nodes express CXCR3 (**p< 001; $* \star \star p<0.001)$. (G) Flow plot of PD-1+ CXCR5+ (GC) Tfh cells shows AIM+ cells following stimulation with spike; scatter plot shows specificity of GC Tfh cells to SARS-CoV-2 spike and nucleocapsid. The dashed line represents undetectable responses assigned a value of $0.01 \%$. Black squares denote SARS-CoV-2 unexposed animals.
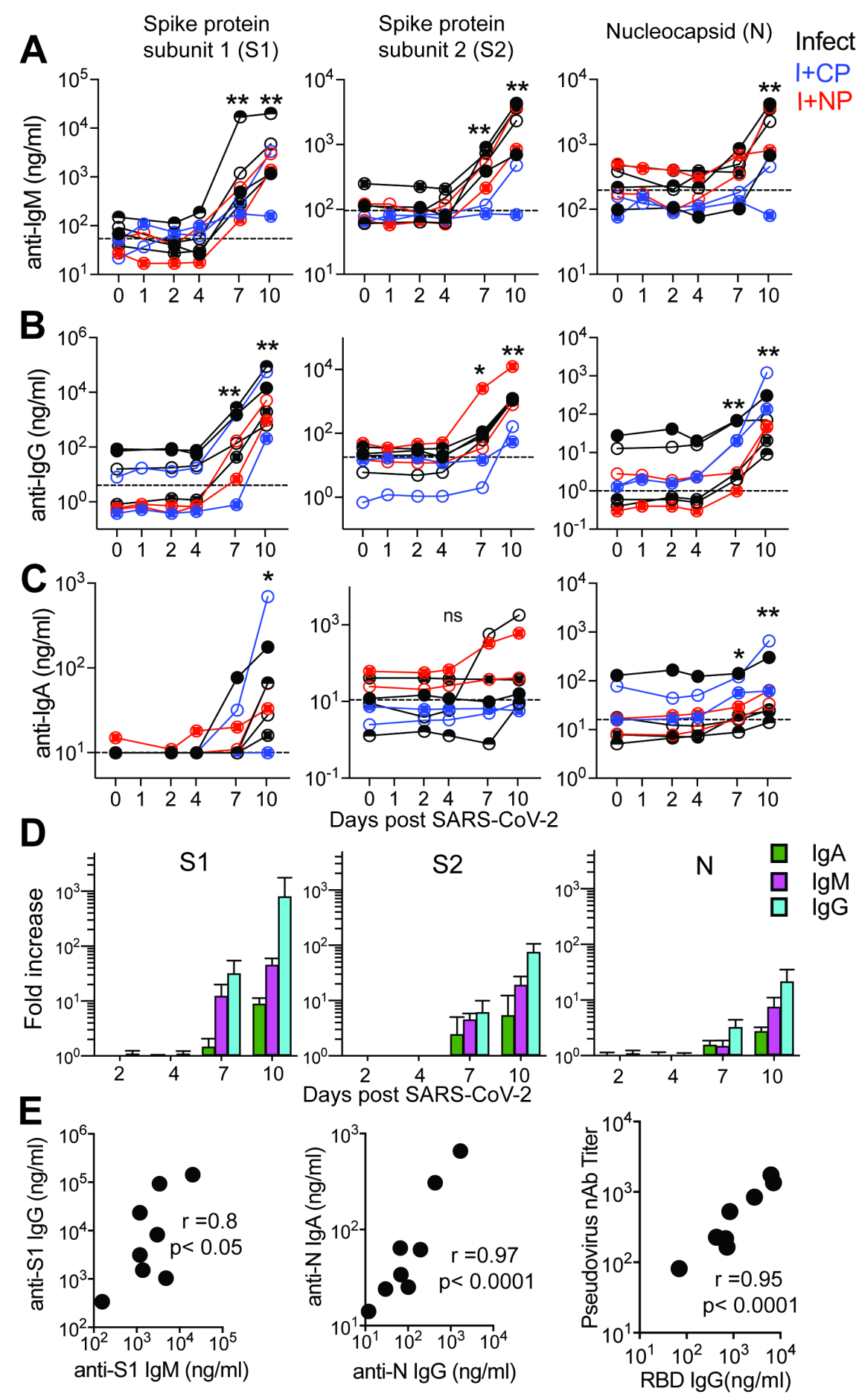

Figure 4

Figure 4 
Humoral responses to SARS-CoV-2 are dominated by $\lg$ G antibodies Concentrations of (A) $\lg M$, (B) $\lg$, and (C) IgA antibodies specific for S1, S2, and N proteins measured by BAMA or ELISA in serum. The dashed line represents the median pre-infection (day 0 ) concentration for all animals. (D) Fold increase in antibody responses in animals not given $\mathrm{CP}$ was determined by dividing post-infection concentrations by those measured on day 0 in each animal. Geometric mean fold increases with SEM are shown. (E) Correlations between day 10 levels of S1-specific $\lg G$ and $\lg M, N$-specific $\lg A$ and $\lg G$, and pseudovirus neutralizing antibody titers and anti-RBD IgG antibodies measured by ELISA. Unique symbols identify animals in each of the experimental groups.

\section{Supplementary Files}

This is a list of supplementary files associated with this preprint. Click to download.

- 20200706CoVSupplementarymaterial.docx

- Figures1.png

- Figures2.jpg

- FigureS3.png

- Figures4.png

- Tables1.png

- Tables2.png

- TableS3.png 